Multivariate analysis for all risk factors proved statistically insignificant.

Abstract 1660 Table 1 Multivariate risk stratification

\begin{tabular}{llcccc}
\hline Factors & Group & Patients & Procedures & Mortality & p value \\
\hline Age (months) & $<1$ Bet 1-12 $>12$ & 61811 & 82114 & 131 & 0.79 \\
Weight $(\mathrm{kg})$ & $<2.52 .6-55.1-10>10$ & 312128 & 3141511 & 0311 & 0.51 \\
Sex & Male Female & 2114 & 2716 & 32 & 0.89 \\
Time bet surg/ & $<11-3$ 3-5 5-7 $>7$ & 346518 & 377521 & 01112 & 0.69 \\
Inter (days) & & & & & \\
Material & Native Synthetic & 276 & 356 & 41 & 0.80 \\
\hline
\end{tabular}

Abstract 1660 Table 2 Multifactorial comparison between mortality and survival

\begin{tabular}{llcccc}
\hline Factors & Group & Mean & SD & Range & p value \\
\hline Age (months) & Mortality Survival & 11 & 14.6 & $0.3-36$ & 0.792 \\
& & 21.2 & 40.9 & $0.3-192$ & \\
Weight (kg) & Mortality Survival & 6.6 & 6.3 & $2.7-17.5$ & 0.513 \\
& & 7.3 & 5.8 & $1.9-33.9$ & \\
Time (days)bet & Mortality Survival & 7.8 & 5.4 & $3-17$ & 0.399 \\
surg/intervention & & 13.5 & 14.8 & $1-59$ & \\
\hline
\end{tabular}

Conclusion We conclude that transcatheter intervention in critically sick patients during immediate post operative period ta safe in expert hands and in a fully equipped facility, backed up by multi specialty team.

\section{COMPUTATIONAL FLUID DYNAMICS SIMULATION OF PRESSURE CHANGES IN THE TRACHEA BEFORE AND AFTER VASCULAR RING SURGERY: THE FEASIBILITY ANALYSIS}

doi:10.1136/archdischild-2012-302724.1661

${ }^{1} \mathrm{FL}$ Chen, ${ }^{2,3} \mathrm{TC}$ Shih, ${ }^{4} \mathrm{TL}$ Horng. 'Division of Pediatric Cardiology, Chung Shan Medical University Hospital; '2Department of Biomedical Imaging and Radiological Science, China Medical University; ${ }^{3}$ Department of Radiology, China Medical University Hospital; ${ }^{4}$ Department of Applied Mathematics, Feng Chia University, Taichung, Taiwan R.O.C.

Background Effective relief of tracheal stenosis (TS) caused by complete vascular ring (CRV) is mandatory for vascular ring surgery (VRS).Even the forced expiratory volume in the first second (FEV1) measurement is usually applied to assess TS caused by CVR, here has been little research about the quantitative approach for analyzing the airway. Our purpose will apply the computational fluid dynamic (CFD) technique to evaluate the change of tracheal airway pressure by VRS.

Methods 12 patients with CVR and TS were found of pressure drops across the tracheal airway segment of TS before and after the VRS. CFD was performed to obtain the velocity field and viscous pressure drops in a realistic, three-dimensional, patient-specific model. The tracheal aerodynamic resistance was represented as a pressure drop in the tracheal airway. Three velocities (0.01, 0.1 , and $1 \mathrm{~m} / \mathrm{s}$ ) were used to calculate the pressure drop in the tracheal airway for both inspiratory and expiratory flow patterns.

Results The pressure drops of the TS before and after the VRS at inlet velocity $0.1 \mathrm{~m} / \mathrm{s}$ was improved $45.95 \%$ in inspiratory phase and $40.65 \%$ in expiratory phase. When the inspiratory inlet velocity reached $1 \mathrm{~m} / \mathrm{s}$, the pressure drop became improved of $43.32 \%$. CFD showed a surgical treatment can significantly decrease the pressure drop in the tracheal airway, especially in a low inlet velocity.

Conclusion CVR can augment the airway flow resistance of TS. The CFD approach can be a useful alternative for quantifying the change of airway resistance and evaluating the effectiveness of VRS.
OUTCOME OF COGNITIVE PERFORMANCE IN SCHOOL-AGED CHILDREN AFTER SURGICAL CORRECTION OF CONGENITAL VASCULAR RING

doi:10.1136/archdischild-2012-302724.1662

${ }^{1} \mathrm{FL}$ Chen, ${ }^{2} \mathrm{ML}$ Yang, ${ }^{3} \mathrm{YC}$ Li. 'Division of Pediatric Cardiology, Chung Shan Medical University Hospital; ${ }^{2}$ College of Medicine, Department of Anatomy; ${ }^{3}$ College of Medicine, Department of Pharmacology, Chung Shan Medical University, Taichung, Taiwan R.O.C

Objective Our goal in this study is to assess the impact of congenital vascular ring (VR) on cognitive performance and its outcome after surgical correction.

Background The clinical indication for VR abnormalities relies on symptoms of tracheal and esophageal compression. However, the possible influence of VR on neurocognitive function and intelligence in school-aged children has yet to be examined.

Methods Patients with VR ( $\mathrm{n}=78)$, which divided into two groups, partial (PVR) or complete vascular ring (CVR), and 30 normal controls were analyzed with Wechsler Intelligence Scale for Children-III (WISC-III) to assess the intellectual abilities before and one year after surgical intervention.

Results The initial assessment showed that the overall performances of the two VR groups on all the intelligence measurements are significantly lower than the control group. Nevertheless, the post-operational assessment of the VR groups one year later had shown significant improvement in most of the intelligence categories measured.

Conclusions The current results indicate a strong possibility of the influence of VR on neurocognitive development in school-aged children which is prompt to improve after surgical intervention is applied.

\section{EARLY CORONARY FLOW AND ECG CHANGES FOLLOWING CARDIOPULMONARY BYPASS SURGERY IN CHILDREN WITH CONGENITAL HEART DISEASE}

doi:10.1136/archdischild-2012-302724.1663

${ }^{1}$ EH Aburawi, ${ }^{2 P}$ Liuba, ${ }^{2} \mathrm{E}$ Pesonen. 'Paediatrics, UAE University and Lund University, Al Ain, United Arab Emirates; 'Lund University and Skåne University Hospital Lund, Lund, Sweden

Background Surgery with cardiopulmonary bypass (CPB) remains the mainstay of therapy in children with congenital heart defects but little is known about its pathophysiologic consequences. We have recently demonstrated that CPB surgery in children leads to increase in coronary flow for at least 1 week after surgery, while others have shown, yet in adults, profound adverse effects of CPB on myocardial repolarization over the same period of time. The latter may be an important mechanism of ventricular arrhythmia, which is a common complication after CPB surgery.

Objective To investigate the relationship between coronary flow and myocardial repolarization after CPB surgery.

Methods Coronary flow in the proximal part of the LAD and indexes of myocardial repolarization (QT interval corrected for heart rate (QTc) and $\mathrm{QT}$ dispersion) were assessed by transthoracic Doppler echocardiography and in 12-lead surface electrocardiogram, respectively, in children with atrial $(n=12)$ and atrioventricular septal defects $(n=16) 1$ day before, and 5 days after cardiac surgery with $\mathrm{CPB}$.

Results Neither QTc nor QT dispersion postoperatively differed significantly compared with preoperative values ("p for mean" $>0.2$ for both). However postoperative QTc showed a significant positive correlation with both preoperative $(\mathrm{r}=0.4, \mathrm{p}=0.03)$ and postoperative $(r=0.5, p=0.01)$ coronary flow. In patients with atrial septal defects, OTc postoperatively correlated significantly with the duration of CPB ( $r=0.6, p=0.04)$, but not in those with VSD. 
Conclusion In a small cohort of children undergoing CPB surgery, increased coronary flow and, to less extent, prolonged CPB appeared to be associated with heightened risk for delayed myocardial repolarization after surgery.

\section{IMPACT OF CHYLOTHORAX ON THE POST OPERATIVE OUTCOME AFTER PEDIATRIC CARDIOVASCULAR SURGERY}

doi:10.1136/archdischild-2012-302724.1664

SR Ismail, M Kabbani, H Najm, G Shaath, A Jijeh. Cardiac Science, King Abdulaziz Medical City for National Guards, Pediatric Cardiac Intensive Care, Riyadh, Saudi Arabia

Background Chylothorax is the accumulation of Chyle in the pleural cavity, usually develops after disruption of the thoracic duct along its intra-thoracic route. In the majority of cases this rupture is secondary to trauma (including cardio thoracic surgeries). Chylothorax is a potentially serious complication after cardiovascular surgeries that require early diagnosis and adequate management.

Methods A retrospective study of all cases Complicated with Chylothorax after pediatric cardiac surgery in King Abdulaziz Cardiac Center between January 2007 and December 2009 was conducted. The study aim to determine the risk factors, and the impact on the post operative course.

Results We have 1135 cases operated during the study period, 57 cases (5\%) were complicated with chylothorax in the post operative period, The most common Surgeries complicated with chylothorax were the single ventricle repair surgeries (Glenn- Fon$\tan ) 15$ cases (27\%), followed by the arch repair cases 10 cases $(18 \%)$, the ventricular septal defect cases 10 cases (18\%), the Atrioventricular septal defect cases 7 cases (12\%), the arterial switch cases 6 cases $(11 \%)$, and others 8 cases (14\%). The ICU stay, the length of hospital stay and the bypass time were significantly longer in the chylothorax group, also the ventilation time, the inotropes duration and number were higher in the chylothorax group.

Conclusion Chylothorax after pediatric open heart surgery is not an uncommon complication, it Occurs more commonly with single ventricle repair and Aortic arch repair surgeries, it has a significant impact on the post operative course and post operative morbidity.

\section{NONINVASIVE MONITORING OF HEMOGLOBIN CONCENTRATION IN PEDIATRIC CARDIAC POSTOPERATIVE PATIENTS}

doi:10.1136/archdischild-2012-302724.1665

P García Soler, JM Camacho Alonso, A Morales Martínez, G Milano Manso. Pediatric Intensive Care Unit, Hospital Regional Universitario Materno Infantil Carlos Haya, Málaga, Spain

Background/Aim One of the major concerns after cardiovascular surgery is to detect bleeding and optimize hemoglobin $(\mathrm{Hb})$ to improve cardiac output, leading to repeated blood tests. We describe our experience with a new method of a noninvasive and continuous measurement of $\mathrm{Hb}$ concentration.

Methods Partial results of a prospective study in children after cardiac surgery, from January to March 2012. SpHb monitoring was performed during the first 48 hours after surgery (Pulse Co-Oximeter Radical-7TM/7.8.0.1, Masimo, CA). When each blood sample was taken, we collected the data from Radical- $7^{\mathrm{TM}}$, using central laboratory as a reference method (SiemensADVIA2120i). Data are reported as mean values and SD (normally distributed) and as median values and minimum-maximum range (distribution non-normal).

Results 78 blood samples were drawn from 21 patients, with a median age of 1.3 months (0.23-56), a median weigh of $3.7 \mathrm{~kg}$ (3-16.6) and a perfusion index(PI) of $1.2(0.21-13)$. All the measurements were performed in sedated subjects, $79.5 \%$ of them were intubated. Mean $\mathrm{Hb}$ on the laboratory analyzer was $12.47 \pm 2.28 \mathrm{~g} / \mathrm{dl}$ and mean $\mathrm{Hb}$ on the pulse oximeter $(\mathrm{SpHb})$ was $13.2 \pm 2.26 \mathrm{~g} / \mathrm{dl}$; its correlation coefficient was 0.75 and $\mathrm{R}^{2}$ was $0.55(\mathrm{p}<0.05)$. The mean of differences between both methods was $0.75 \pm 1.6 \mathrm{~g} / \mathrm{dl}$. BlandAltman plot shows that $65 \%$ had a difference $<1 \mathrm{~g} / \mathrm{dl}$ in comparison with laboratory hemoglobin.

Conclusions $\mathrm{SpHb}$ offers moderately acceptable accuracy in pediatric cardiac postoperative patients, maybe influenced by the low weight and PI of our patients. It has the advantages of providing continuous measurements, noninvasively, which may facilitate hemoglobin monitoring in the intensive care unit.

\section{INCIDENCE AND RISK FACTORS FOR POST CARDIAC SURGERY ARRYTHMIAS IN A TERTIARY PAEDIATRIC CARDIAC REFERRAL CENTRE}

doi:10.1136/archdischild-2012-302724.1666

${ }^{1} \mathrm{~K}$ Adhvaryu, ${ }^{2} \mathrm{R}$ Johnson, ${ }^{1} \mathrm{~N}$ Shetty. ${ }^{1}$ Paediatric Intensive Care Unit; ' ${ }^{2}$ Department of Cardiology \& Cardiothoracic Surgery, Alder Hey Children's Hospital, Liverpool, UK

Background and Aims Arrhythmias in the immediate postoperative period are a significant cause of morbidity. Its incidence varies between $15-79 \%$ and depends on case mix of surgical patients and methods used to identify them. Our aim was to determine the incidence and risk factors for their occurrence in our regional cardiac referral centre.

Methods A retrospective study was conducted including all paediatric (newborn to 18 years) patients who underwent a surgical procedure requiring cardio-pulmonary bypass between September 2010 and August 2011. Patients were identified and data gathered from the cardiac databases, electronic patient records and typed operation notes. Patients were excluded if they had pre-operative arrhythmias or if the arrhythmias during surgery were so transient such that they only occurred in the operation theatre.

Results Of the 346 cases that met inclusion criteria, 15\% developed arrhythmias of which half required intervention. Risk factors for arrhythmias included surgery involving the septum (21\% vs $8 \%$ non-septal), male (17\% vs $14 \%$ ) and age less than one year (20\% vs $9 \%)$. Those with arrhythmias had significantly longer bypass (160min vs $116 \mathrm{~min}, \mathrm{p}<0.05)$ and cross clamp times (101 min vs $77 \mathrm{~min}, \mathrm{p}<0.05)$. Transient rhythm problems that required intervention were sinus bradycardia, junctional rhythm, junctional ectopic tachycardia and supra ventricular tachycardia, whilst $43 \%$ of those with heart block required permanent pacing.

Conclusion Despite advances in surgical techniques arrhythmias after cardiac surgery were seen in a significant number $(15 \%)$ of our patients although only a small proportion $(<1 \%)$ require long term interventions.

\section{PERIOPERATIVE CHANGES IN RENAL BIOMARKERS IN CHILDREN UNDERGOING OPEN HEART SURGERY}

doi:10.1136/archdischild-2012-302724.1667

J Krastins, Z Straume, J Auzins. Pediatric Intensive Care, University Children's Hospital, Riga, Latvia

Introduction Cardiac surgery with cardiopulmonary bypass (CPB) is commonly perceived as a risk factor for decline in renal function. Hypothermia, hypoxia, hypotension, non-pulsatile blood flow during $\mathrm{CPB}$, use of $\mathrm{ACE}$ inhibitors, inotropic and (or) vasoactive support affects kidney and contributes to the acute kidney injury (AKI)

Objective To evaluate dynamics of renal biomarkers - serum creatinine (SCr) and glomerular filtration rate (GFR) in children undergoing open heart surgery.

Methods We conducted prospective, non-randomized observational study at the tertiary care University Children's Hospital Pediatric ICU. We enrolled 30 patients, 12 boys and 18 girls with CHD. Their median body weight was $6.8 \mathrm{~kg}$, (IOR $5.2<8.2 \mathrm{~kg})$ and median 